\title{
Time-delay Compensation Using Energy Tank for Satellite Dynamics Robotic Simulators
}

\author{
Marco De Stefano ${ }^{1}$, Luca Vezzadini ${ }^{2}$ and Cristian Secchi ${ }^{2}$
}

\begin{abstract}
In this work we present a novel approach which compensates the destabilising effects of the time delay intrinsic in the control loop of an admittance-controlled robot employed for satellite dynamics simulation. The method is based on an energy storing element, the tank, which is exploited by the controller to preserve the passivity of the system and to avoid instability. Furthermore, we compare the performance of the proposed method with existing energy-based approaches, namely time-domain-passivity and wave variable transformation. The performance comparison and robustness of the methods are analysed in a Montecarlo simulation and validated experimentally.
\end{abstract}

\section{INTRODUCTION}

A key role for the success of an OOS (On-Orbit Servicing) space mission is played by the validation of the algorithms and controls prior to the launch. Hence, having a reliable simulator, which can reproduce micro-gravity conditions on ground is of utmost importance. Within this context, several technologies can be adopted to recreate zero gravity conditions on ground and these can be classified into: air bearing systems, neutral buoyancy, 0-g parabolic flights, cable offloaders and hardware-in-the-loop simulators [1].

In simulation with hardware in the loop, a computer-based dynamic model of the whole space system is considered and a hardware system, capable of delivering a six dimensions Cartesian motion, is used to reproduce the desired behaviour. Usually, the simulator which exploits this technology is a robot(s) equipped with a force-torque sensor at the endeffector and a satellite mock-up [2]. The force-torque sensor is used to measure external interaction.

In recent years, such kind of simulators have been employed in the aerospace field and there are several facilities exploiting this technique available in literature. The Special Purpose Dexterous Manipulator (SPDM) facility is composed of two arms with a total of 15 degrees of freedom (dof) and it is employed for verification of robotic tasks performed on the international space station [3]. The Lockheed Martin SOCS (Space Operation Simulation Center) facility is composed of two robots with six degrees of freedom each capable of simulating a full-scale spacecraft motion relative to an other object [4]. EPOS (European Proximity Operation Simulator) is composed of two industrial robots, which can be used to simulate rendezvous and docking [5]. INVERITAS is a robotic facility used for the simulation of rendezvous and capture of satellites [6].

\footnotetext{
1 The author is with the Institute of Robotics and Mechatronics, German Aerospace Center (DLR), 82234 Wessling, Germany.

2 The authors are with the University of Modena and Reggio Emilia, 41100 Modena, Italy. Contact: marco.destefano@dlr.de
}

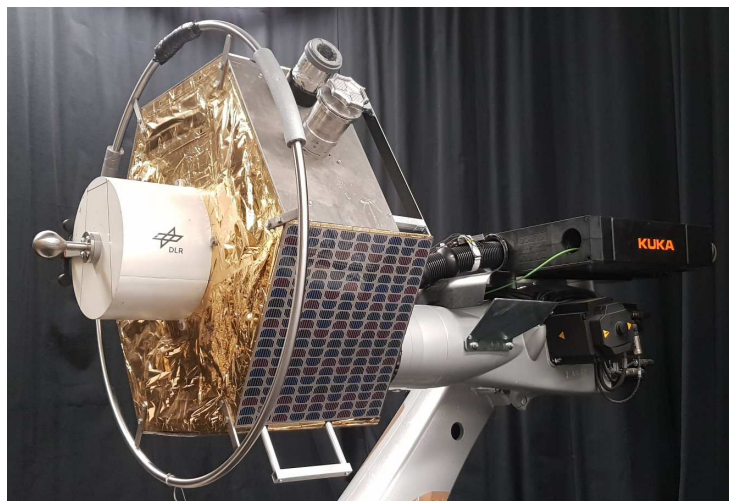

Fig. 1: Client robot of the OOS-Sim facility exploited for satellite dynamics simulation. The industrial robot is equipped with a forcetorque sensor at the end-effector.

An other example is the OOS-Sim facility, which is composed of two industrial robots employed for testing a complete on-orbit servicing task (e.g. grasping of a satellite) on ground [7]. One of the robots is shown in Fig. 1 and it is equipped with a force-torque sensor exploited for satellite dynamics simulation under interaction forces. The force and torque signals measured by the sensor represent the input to the model-based dynamics whose output is commanded to the robot that moves in Cartesian space. However the time delay from a hardware contact (measured by the sensor) to the corresponding simulation driven reaction is recognised as a limitation for the robotic simulators when they are employed for satellite dynamics simulation [2]. In particular, the time delay in the control loop might lead the system to become unstable [8], [9].

The field of teleoperation has exploited approaches based on passivity to deal with instabilities due to time delay in communication channels [10]. Three popular and effective approaches are time domain passivity approach (TDPA), wave variables (WV) and energy tanks [11]. The TDPA is based on an energy observer and a passive controller to compensate the effect of the time delay [12]. In the context of satellite simulation using robots, [13] and [14] extend the TDPA treatment for an admittance controlled robot emulating the dynamics of a satellite. In [15] and [16], the effects due to the discrete-time integration are compensated exploiting the TDPA method. Related to the wave variable transformation, [17] proposes a passivity-based formalism which allows one to guarantee stability and energy conservation for teleoperation system with time delay. The concept of wave variable was generalised in [18] for mechanical and robotic systems. 
In [19] the wave variable transformation was exploited to modulate the energy and improve the transparency of a teleoperation system. Regarding the energy tank, [20] proposes an impedance control with time-varying stiffness to guarantee system passivity. For admittance controlled robots, [21] exploits the tank for controlling a robotic arm for surgical application and [22] extends the method considering interactions with the environment. However, energy tank methods have not been applied on admittance control robot used for free-floating dynamic simulation. Furthermore, although TDPA, energy tank and wave variable represent three of the main control actions that can be found in literature, very few works related to the comparison have been proposed. Quantitative studies comparing the performances of several bilateral control methods can be found in [23] and [24]. In [25] a comparison of the performance using TDPA and wave variable transformation was performed. However the energy tank method was not considered and the analysis was performed only for a time-delayed teleoperation system.

The contribution of this paper is threefold. First, we design a method based on an energy tank to compensate the time delay in the control loop of an admittance-controlled robot exploited for the simulation of satellite dynamics. Second, the performance and robustness of the method are compared with two other existing energy-based methods (TDPA and WV) in a Montecarlo simulation. Third, we validate the methods on a real robot.

The paper is structured as follows. Sec. II introduces the problem statement and Sec. III provides a background on the common energy-based method (TDPA and wave variables) which will be considered for the comparison. The proposed method is described in Sec. IV. Results and comparison with the other methods are presented in Sec. V with experiments ${ }^{1}$ in Sec. V-B. The robustness analysis is performed in Sec. VI and Sec. VII concludes the paper.

\section{Problem Statement}

Industrial robots can be controlled only by means of position commands and this factor imposes an admittance causality on the controller architecture for rendering the dynamics of a satellite. In this case, the model-based dynamics of the satellite provides desired velocity set-points to the robot and it moves in Cartesian space.

We assume that the industrial robot can perfectly track the desired velocity set-points. This is a common assumption and it can be achieved by properly tuning the gains of the low level controllers [26]. However, the internal control of the robot and the inverse kinematics calculation might require several sampling steps for the computation of the desired set-point. Therefore, these factors introduce time delays in the corresponding motion of the robot [13].

A schematic of the industrial robot used for rendering the satellite dynamics is shown in Fig. 2 where the time delay, $T D$, is located in the control loop and it represents

\footnotetext{
${ }^{1}$ Experiments related to the control based on energy-tank can be seen also in the video accompanying the paper.
}

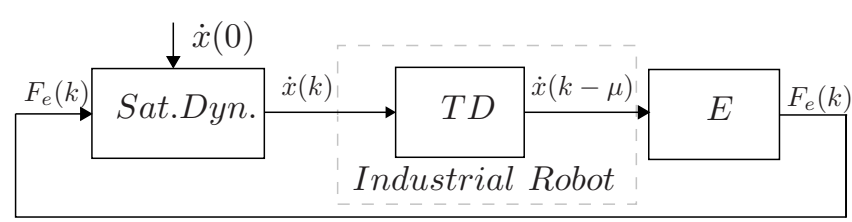

Fig. 2: Admittance model of the robotic simulator. Sat.Dyn is the model-based dynamics, $T D$ is the robot seen as time delay in the loop and $E$ is the environment.

the robot. The robot receives the velocity signals, $\dot{x}(k)$, from the satellite dynamics model (later defined) and its resulting velocity, $\dot{x}(k-\mu)$, is delayed by a quantity $\mu$, where $\mu$ is the number of discrete-time steps of sampling time $T_{s}$. Note that both variables are available, $\dot{x}(k)$ being the commanded velocity and $\dot{x}(k-\mu)$ the measured one.

The commanded velocity value is provided by the satellite dynamics, represented by the block Sat. Dyn in Fig. 2. In the analysis we will consider the translational dynamics represented by the following equation,

$$
\ddot{x}=M^{-1} F_{e},
$$

where, $\ddot{x} \in \mathbb{R}^{3}$ is the acceleration, $M \in \mathbb{R}^{3 \times 3}$ is the mass matrix and $F_{e} \in \mathbb{R}^{3}$ is the external measured force. Therefore, the commanded velocity, $\dot{x}(k) \in \mathbb{R}^{3}$ is obtained by integration of (1). The block $E$ represents the environment with which the robot can interact and the force $F_{e}$ is the external force vector, which can be measured using a force sensor.

The effect of the time delay in the control loop (for the scenario shown in Fig. 2) can be seen in the following example. Let us consider a rigid-body motion described by (1) which collides against two virtual walls, where the generated forces are modelled as spring-damper system. Fig. 3 (top) compares the velocity $\dot{x}_{i}$ in the ideal case (no time delay), with the velocity where a time delay of $20 \mathrm{~ms}$ is located in the loop, i.e. $\dot{x}_{t d}$. As can be seen, the velocity increases its magnitude after each contact and the system might become unstable. Furthermore, the simulated
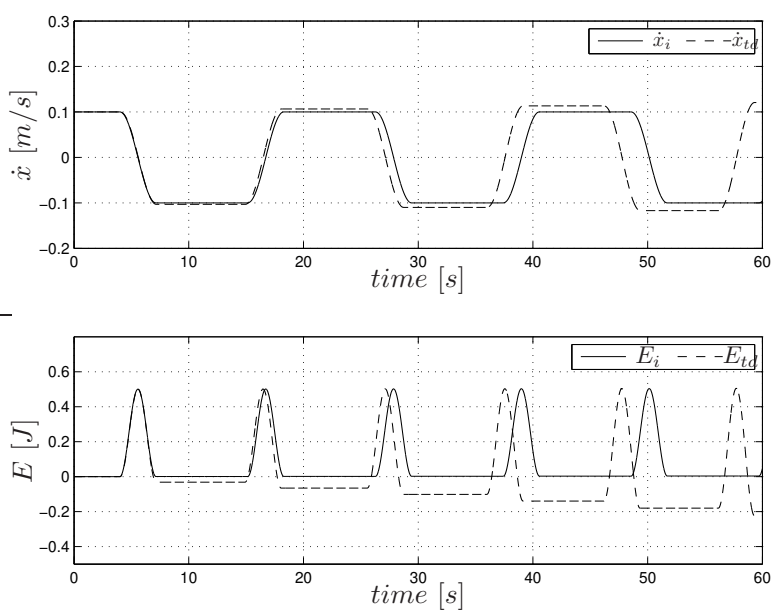

Fig. 3: Velocity and energy comparison. Increase in velocity and energy due to the time delay in the loop. 
motion results to be physically inconsistent. Indeed, the delay introduces extra energy in the system, as can be seen in Fig. 3 (bottom) which compares the energy in the ideal case, $E_{i}$, and the energy with the time delay in the loop, $E_{t d}$.

The increase in velocity and in energy causes an unstable behaviour to the robotic simulator and an energy inconsistent motion. In the following section passivity techniques are introduced to deal with this phenomenon.

\section{PAssivity AND Time-DElay Compensation METHODS}

In this section the concept of passivity is introduced and a background on the methods, which can compensate the time delay in the loop is provided. In particular, the considered methods are Time Domain Passivity Approach (TDPA), which was proposed in [13] and the wave variable transformation [17]. These two methods will be compared with the proposed control, later presented in Sec. IV.

\section{A. Passivity property}

Passivity is a sufficient condition for achieving stability [27] and it is based on input/output characteristic of a system. The passivity condition for a generic system with power port $(F(k), \dot{x}(k)) \in \mathbb{R}^{n}$ can be expressed as in [28, §2] and it is reported below,

$$
E(m)=E(0)+\sum_{k=0}^{m} \dot{x}^{T}(k) F(k) T_{s} \geq 0,
$$

where $E(0)$ represents the initial energy stored in the system and $T_{s}$ is the sampling time. Equation (2) states that the system can not produce energy more than its initial storage and the input energy. Otherwise the system results to be active, i.e. $E(m)<0$, and it will produce energy which can destabilise the system [29].

The presence of time delay in the loop breaks the passivity of the system because it introduces extra energy. In particular this can be seen in Fig. 3 (bottom) where the presence of time delay in the loop violates the passivity condition given in (2), (see the negative trend of energy $E_{t d}$ ).

\section{B. Control Methods for Time-delay Compensation}

The compensation of the time delay has been tackled in teleoperation where usually the control of a remote robot is affected by delays in the communication channel [10]. A popular method to compensate the lag in the signals is based on wave variable transformation. Other existing methods are available and these are based on the passivity characteristic of the system, namely energy-tank and TDPA.

1) TDPA: This methods relies on two main elements, a Passivity Observer (PO) which monitors the energy in the system and a Passivity Controller (PC) which acts when passivity property given in (2) is violated. The passivity observer can monitor the energy injected by the time delay in the system. For the case shown in Fig. 2, the energy observer is defined as,

$$
\begin{array}{r}
E_{o b s}(k)=E_{o b s}(k-1)+F_{e}(k)^{T}(\dot{x}(k)-\dot{x}(k-\mu)) T_{s} \\
+\beta(k-1) F_{e}(k-1)^{T} F_{e}(k-1) T_{s},
\end{array}
$$

where the second term on the right side is the energy due to the time delay of the network and the last term, the energy due to the passivity controller, later defined.

The passivity controller is based on an admittance causality with a variable damping factor $\beta$, which varies as a function of the observed energy flow. In the admittance configuration, the velocity is modified to produce the dissipation and a corrected velocity value is commanded to the robot as follows,

$$
\dot{x}_{c}(k)=\dot{x}(k)-\underbrace{\beta(k-1) F_{e}(k)}_{\dot{x}_{p c}(k)},
$$

where $\beta(k-1)$ is the time varying damping factor enabled when the passivity condition is violated and it is given as,

$$
\beta(k)= \begin{cases}-\frac{E_{o b s}(k)}{F_{e}(k)^{T} F_{e}(k) T_{s}} & \text { if } E_{o b s}(k)<0 \\ 0 & \text { else. }\end{cases}
$$

Note that the time-varying damping is free of mathematical singularity because it acts only when the $F_{e} \neq 0$.

Therefore, the final velocity sent to the robot, $\dot{x}_{c}$, will be modified according to the time-varying damping. Thus the system with the passive control action is rendered to be stable [13]. Fig. 4 shows the architecture of the robotic simulator endowed with the PO-PC architecture. The $P O$ is defined in (3) and the $P C$ is the time-varying damping in (5). Finally, the robot receives a corrected velocity $\dot{x}_{c}$ given in (4).

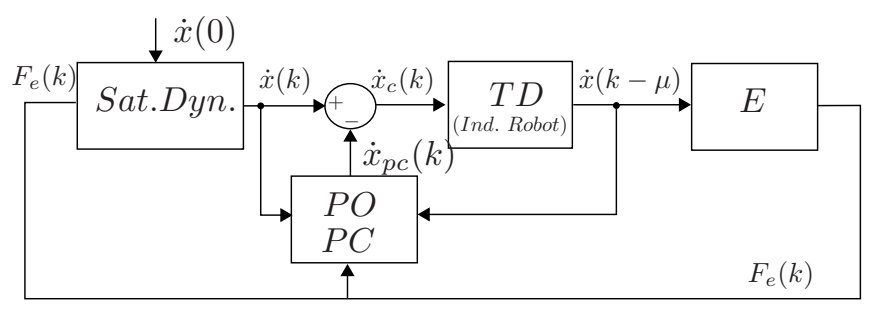

Fig. 4: Admittance model of the robot simulator with time delay (TD) in the loop and PO-PC architecture.

2) Wave variable: Wave variable is a common method utilised in teleoperation [30] for time delay compensation. In particular, it is based on a simplified transformation of the standard variables $\left(\dot{x}, F_{e}\right)$ to the wave variables $(u, v)$, where $u$ is the forward wave, from master to slave, and $v$ the returning wave, from the slave to master. The model described in Fig. 2 can be augmented with the wave variable transformation and it is shown in Fig. 5.

The wave variable layers $(W V)$ introduced in the scheme compute the wave characteristics defined as follows:

$$
\begin{aligned}
& u_{l}=\frac{b \dot{x}(k)+F_{W V}(k)}{\sqrt{2 b}}, \\
& v_{l}=\frac{F_{W V}(k)-b \dot{x}(k)}{\sqrt{2 b}}, \\
& u_{r}=\frac{F_{e}(k)-b \dot{x}(k-\mu)}{\sqrt{2 b}}, \\
& v_{r}=\frac{b \dot{x}(k-\mu)+F_{e}(k)}{\sqrt{2 b}},
\end{aligned}
$$




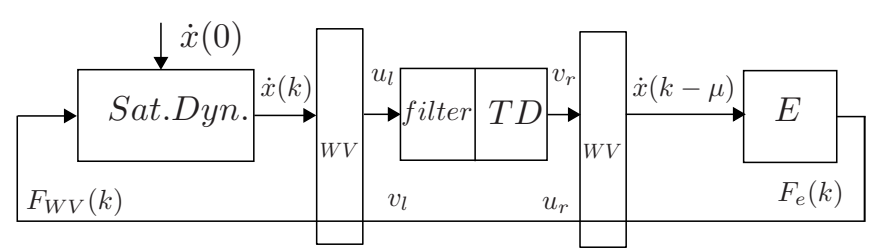

Fig. 5: Admittance model of the robot simulator with time delay (TD) in the loop and wave variable transformation.

where $u_{l}$ and $u_{r}$ are the input waves of the respective port and $v_{l}$ and $v_{r}$ are the output waves. The parameter $b \in \mathbb{R}^{+}$in (6) is the characteristic impedance and it represents a critical role in determining the system response because it represents a trade off between the velocity and the force.

The set of equations given in (6) is commonly used in wave variable formulation, see [17] and it has been adapted for the considered scenario. However, for the analysed scenario the time delay is only in the forward wave and not in the backward wave. Therefore, the input force to the satellite dynamics in Fig. 5 expressed in wave variable transformation is defined as,

$$
F_{W V}(k)=\sqrt{2 b} v_{l}+b \dot{x}(k) .
$$

It is worth to notice that a low-pass filter is introduced in the control loop shown in Fig. 5. Indeed, the wave variable transformation might be affected by high-frequency noise and a common approach to improve the performance is given by using a filter. The phase lag that the filter might cause is considered within the wave variable transformation layer and therefore does not influence the stability [18].

\section{Passivity Control with Energy Tank APPROACH}

In this section the design of the proposed method which compensates the time delay in the control loop is presented. The method is based on an energy storing element, the tank, which will be used by the controller to maintain the passivity properties. Therefore, we augment the dynamic model in (1) with an energy tank, whose role is to store the energy dissipated by the controlled system. Formally, the augmented dynamics is given by the following,

$$
\left\{\begin{array}{l}
M \ddot{x}-w x_{t}=F_{e} \\
\dot{x}_{t}=-w^{T} \dot{x} \\
y=\left(\dot{x}^{T}, x_{t}\right)^{T}
\end{array}\right.
$$

The term $x_{t} \in \mathbb{R}$ is the state associated with the tank and the energy stored in the tank is given by the following equation,

$$
T\left(x_{t}\right)=\frac{1}{2} x_{t}^{2},
$$

where the energy $T\left(x_{t}\right)$ needs to be initialized with a value $x_{\mathrm{t}}(0)>0$ in order to avoid singularities.

The parameter $w \in \mathbb{R}^{3}$ is the control input (later defined) through which is possible to control the exchange of energy between the main admittance model (the satellite dynamics) and the tank. Thus, the augmented admittance model consists of a mechanical system and a tank energetically coupled through the input $w$. Note that the exchange of energy between the tank and the mechanical system is power preserving, i.e. the energy injected (or extracted) in the tank is the same as the one extracted (or injected) by the system, see [21] for details. Indeed, the total variation of energy in the system, $\dot{W}$, is given by

$$
\dot{W}=\dot{V}+\dot{T},
$$

where $\dot{V}$ is the energy variation of the system and $\dot{T}$ is the variation of energy in the tank. In particular, from

$$
V(x, \dot{x})=\frac{1}{2} \dot{x}^{T} M \dot{x},
$$

it follows that

$$
\dot{V}=\dot{x}^{T} M \ddot{x}=\dot{x}^{T} F_{e}+\dot{x}^{T} w x_{t} .
$$

The variation of energy in the tank results to be,

$$
\dot{T}=x_{t} \dot{x}_{t}=-x_{t} w^{T} \dot{x} .
$$

Then, it is straightforward to calculate $\dot{W}$, as the sum of (12) and (13), to find

$$
\dot{W}=\dot{x}^{T} F_{e},
$$

which means that the system is passive according with (2).

\section{A. Control Input for the energy tank}

The method based on energy tank has the flexibility of choosing any control input [29]. Therefore, two common control inputs are considered for the analysed scenario. The first one is based on a spring-damper behaviour and the second one on a spring behaviour only.

It is worth to notice that the control input $w$ imposes not only a control action, but it is also associated with the state of tank, see (8). Furthermore, it uses the available energy in the tank to guarantee the passivity. In particular, the loss of passivity is generated when an external force is involved in the loop with the time delay (as shown in Sec. II). Therefore when a force acts on the system, the control input $w$ will act. Formally, the control input using spring-damper for the system (8) is defined as,

$$
w=\left\{\begin{array}{lc}
-\frac{1}{x_{t}}(K \tilde{x}+D \dot{\tilde{x}}) & T\left(x_{t}\right)>\varepsilon \\
0 & \text { else }
\end{array}\right.
$$

where $K \in \mathbb{R}^{3 \times 3}$ is the stiffness matrix and $D \in \mathbb{R}^{3 \times 3}$ the damping. The term $\dot{\tilde{x}}$ is the error in velocity between the non-delayed values and the delayed one expressed as,

$$
\dot{\tilde{x}}=\dot{x}(k)-\dot{x}(k-\mu),
$$

and $\tilde{x}$ is the respective variation of position, $\tilde{x}=x(k)-x(k-$ $\mu$ ). The value $\varepsilon \in \mathbb{R}^{+}$in (15) represents the minimum value of the energy in the tank needed to implement the control input in order to avoid singularities in the solution and it needs to be set. The control input presented in (15) can be reduced considering only the spring element as follows,

$$
w= \begin{cases}-\frac{1}{x_{t}} K \tilde{x} & T\left(x_{\mathrm{t}}\right)>\varepsilon \\ 0 & \text { else. }\end{cases}
$$




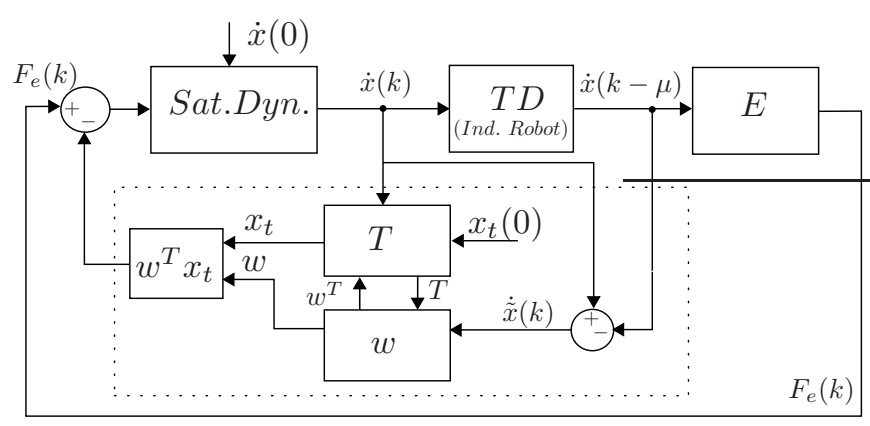

Fig. 6: Admittance model of the robot simulator with time delay (TD) in the loop and energy tank architecture.

Note that the system (8) under the control action given in (15) or (17) results to be passive as demonstrated for a generic input $w$, see (14). The schematic of the system endowed with the energy tank is shown in Fig. 6. The dotted box highlights the elements that belong to the energy tank method. The satellite dynamics receives a correction in force, i.e. $w^{T} x_{t}$, modulated by the tank, $T$, in (9) and the control input $w$ in (15) or (17). The control $w$ (which receives as input the difference in velocity $\dot{\tilde{x}}(k)$ ) is also exploited by the tank in order to store the energy dissipated by the controller.

In the following subsection the results of the two control inputs related to the energy tank method are presented.

\section{B. Results with the Energy Tank Method}

The two control inputs proposed in Sec. IV-A are verified and compared in a simulation study, where the stiffness parameter $K$ is set the same for both controllers. The simulation considers the following conditions.

Example 1: The simulated body has a mass of $100 \mathrm{~kg}$ with an initial velocity of $0.1 \mathrm{~m} / \mathrm{s}$, the time delay in the control loop is $20 \mathrm{~ms}$ and the sampling time is $1 \mathrm{~ms}$. During the motion, the body collides against virtual walls modelled as spring elements in simulation producing forces. These will be substituted by the real force sensor data during the experiments.

Fig. 7 (top) shows the comparison of the velocity using the control input (15), namely $\dot{x}_{1}$ and the control input (17), i.e. $\dot{x}_{2}$. The velocities are also compared with the ideal case $\dot{x}_{i}$. As can be seen the action of the energy tank control removes the destabilising effect discussed in Fig. 3 and the velocity is really close to the ideal one. Fig. 7 (middle) shows the control action given in (15) and (17), which are indicated with $w_{1}$ and $w_{2}$, respectively. The corresponding energies of the tank for the control input (15) and (17) are shown in Fig. 7 (bottom). The presence of more energy in the tank for the control input (15), (see $T_{1}$ in the plot) is given by the presence of the damping term, therefore, more energy is stored in the tank.

The two control inputs behave similarly and the trend of velocity is really close to the ideal case $\dot{x}_{i}$, which considers no delay in the loop. It is worth to compare Fig. 2 (without the proposed method) with Fig. 7 (with the proposed method) in order to understand the benefit of the controller.
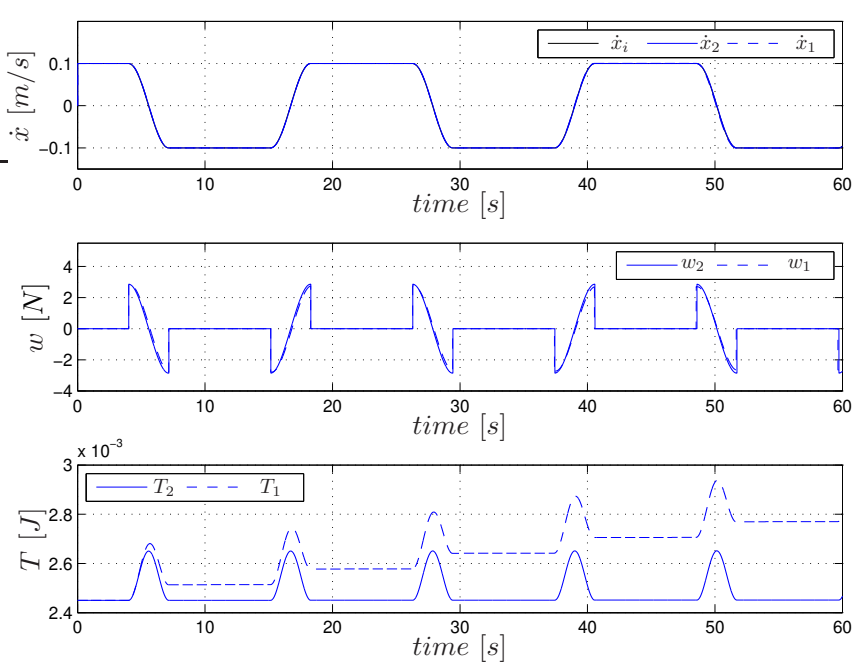

Fig. 7: Top: ideal velocity $\dot{x}_{i}$, velocity $\dot{x}_{1}$ with control input (15) and velocity $\dot{x}_{2}$ with control input (17). Middle: Control input $w_{1}$ given in (15) and $w_{2}$ in (17). Bottom: energy tank with corresponding control input.

Notice that the control input (15) requires the setting of two parameters, namely $D$ and $K$. On the other hand (17) requires only the setting of $K$. For the comparison in the following section, we will consider the control input given in (17) because it reduces the number of parameters obtaining similar performance.

\section{Performance Comparison between the ENERGY-BASED APPROACHES}

In this section, we show the results of simulations and experiments performed with the energy tank, wave variable and TDPA methods. Since the considered dynamics is decoupled, the analysis will be performed for one degree of freedom.

\section{A. Simulation Results}

The proposed controller based on energy tank (see (17) and (8)) is compared with the TDPA and wave variable methods considering the same initial conditions and parameters reported in Example 1.

Fig. 8 shows the velocities of the robot with the TDPA, energy tank and wave variable method, $\dot{x}_{T D P A}, \dot{x}_{\text {etank }}$, $\dot{x}_{W V}$, respectively. Furthermore, the velocities are compared with the ideal case velocity $\dot{x}_{\text {ideal }}$. It is worth to notice how the trend of the velocities is close to the ideal one. However, the velocity produced with the wave variable method is affected by overshoot, as can be seen in the enlarged part of Fig. 8. Furthermore, the velocity produced with the wave variable method is affected also by the presence of noise (see between $0 s$ and $0.1 s$ ).

In order to evaluate the performance between the methods, the mean value for the velocity $\dot{\bar{x}}$ and the energy $\bar{E}$ is calculated for each method and compared with the ideal velocity and energy. Also the Mean Square Error (MSQE) is calculated to see how large is the difference with respect to the ideal dynamics of the satellite. The data are reported in 


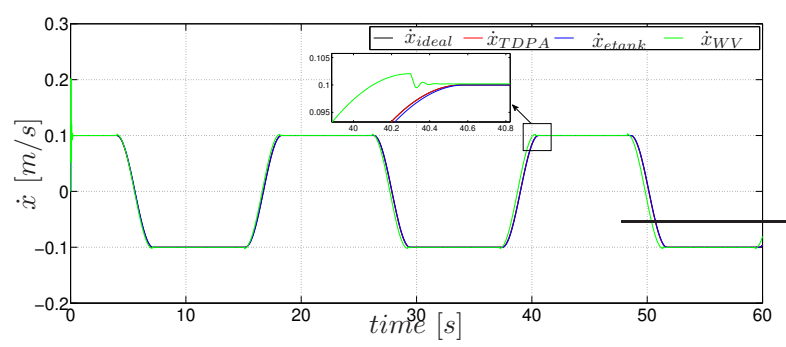

Fig. 8: Velocity comparison between ideal case, $\dot{x}_{\text {ideal }}$, with TDPA, $\dot{x}_{T D P A}$, with energy tank, $\dot{x}_{\text {etank }}$ and wave variable $\dot{x}_{W V}$ method.

\begin{tabular}{|c|c|c|c|c|}
\hline & \multicolumn{4}{|c|}{ Performance } \\
\hline & \multicolumn{2}{|c|}{ Mean Value } & \multicolumn{2}{c|}{ MSQE } \\
\hline & $\dot{\bar{x}}[\mathrm{~m} / \mathrm{s}]$ & $E[J]$ & $\dot{\bar{x}}^{2}\left[\mathrm{~m}^{2} / \mathrm{s}^{2}\right]$ & $E^{2}\left[\mathrm{~J}^{2}\right]$ \\
\hline Ideal & -0.0071 & 0.4345 & - & - \\
\hline TDPA & -0.0071 & 0.4345 & 0.00003 & 0.0001 \\
\hline Energy Tank & -0.0071 & 0.4345 & 0.0001 & 0.0002 \\
\hline Wave Variable & -0.0077 & 0.4365 & 0.0180 & 0.2323 \\
\hline
\end{tabular}

TABLE I: Performance results: mean value and MSQE of the velocity and energy calculated for each energy-based method.

Table I and it can be seen that the velocity of each methods (as well the energy) is closed to the ideal case. In particular, the TDPA and the proposed energy tank method provides the same performance when compared to the ideal case. The slight difference with the wave variable method is due to the overshooting of the velocity after each impact.

\section{B. Experiment Results}

The methods are also experimentally validated on a onedegree of freedom robot, composed of a motor and a torque sensor as shown in Fig. 9.

The facility has a time delay of $20 \mathrm{~ms}$ and the model runs on a $Q N X$ real-time operating system, with $1 \mathrm{~ms}$ of sampling time. The robot is controlled in admittance mode where the set points are provided by the simulated dynamics, thus, it follows the architecture shown in Fig. 2. The rigid bar is attached to the sensor and the range of the free-floating motion is limited by the two physical wall (see Fig. 9) in order to validate the method for rigid impacts. The initial velocity of the simulated mass is $0.17[\mathrm{rad} / \mathrm{s}]$.

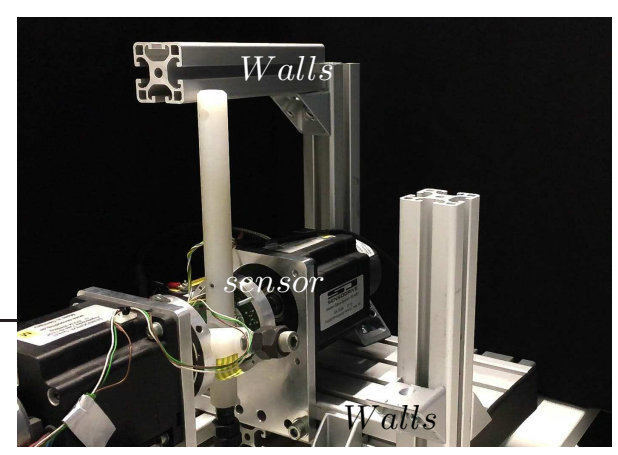

Fig. 9: Experiment set-up: 1 dof robot equipped with a torque sensor. The bar is a mechanical interface which interacts with the walls.
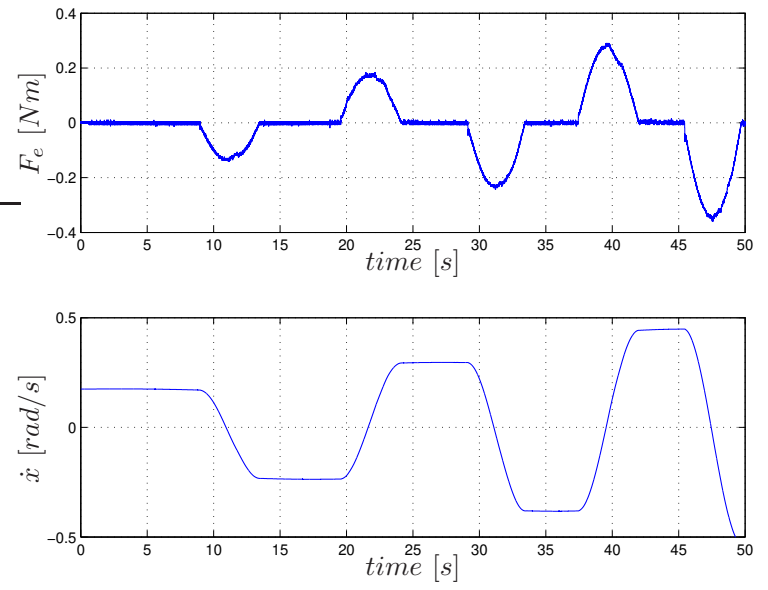

(a) Measured torque (top) and measured velocity (bottom) without energybased control. Unstable system.
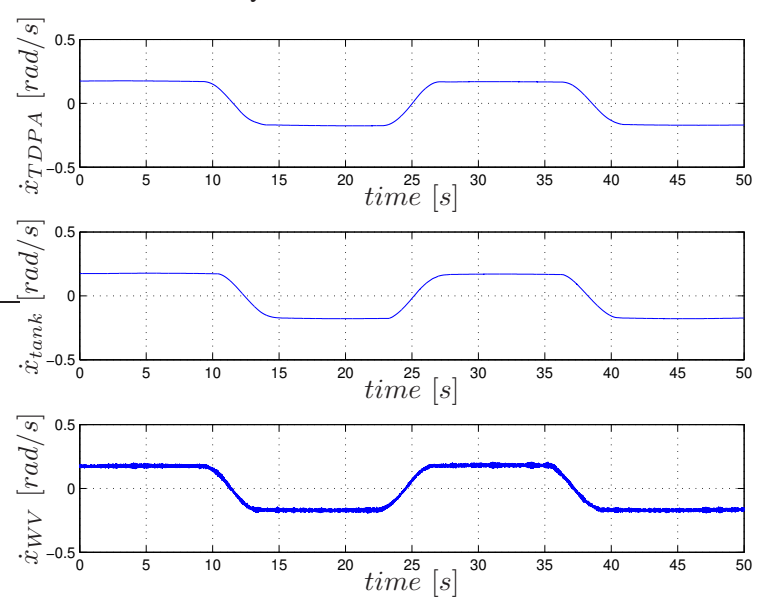

(b) Velocity with the energy-based method. Stable system.

Fig. 10: Experiments results: measured torque and increase in velocity due to time delay (Fig. 10a) and velocity comparison with the energy-based approach in Fig. 10b.

Fig. 10a shows the measured torque during the impact and the relative increase in velocity caused by the time delay when none of the energy-based method is active. As it can be seen the system becomes unstable. When the methods are activated, the passivity of the system is restored and the angular velocity is kept constant after the rigid impact. This is shown in Fig. 10b, which compares the angular velocity for each method. The velocity corrected with the wave variable method, $\dot{x}_{W V}$, is more affected by the noise, which is due to trade off of the characteristic impedance, $b$, and the cut-off frequency of the filter.

The control action, which resolves the unstable behaviour of the system is shown in Fig. 11a for each method. At the top the velocity corrected by the PC is shown, the action of the control input of the energy tank is shown in the middle and the bottom plot shows the control input transformed by the wave variable. Fig. $11 \mathrm{~b}$ shows the energy behaviour with the TDPA method (top) and energy tank (bottom) where the positive trend indicates passivity of the system as per equation (2). 

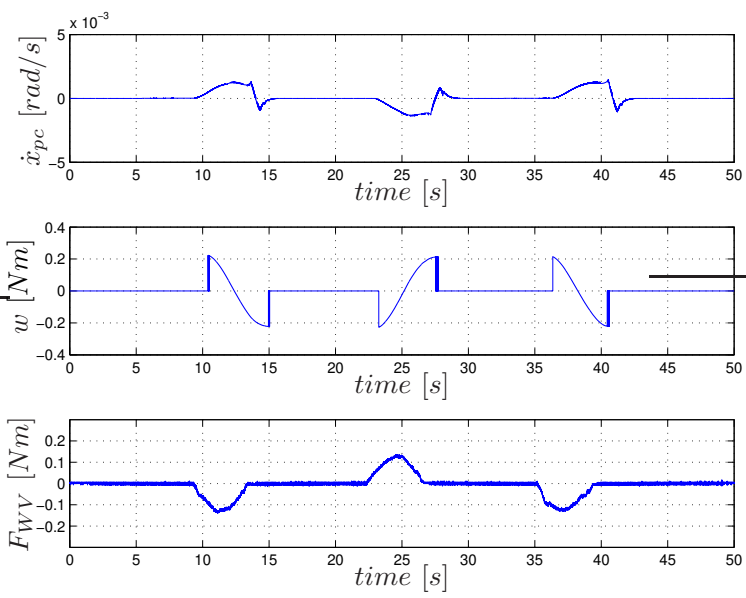

(a) Control input of the TDPA (top), control input of the energy tank (middle) and control input of the wave variable method (bottom).
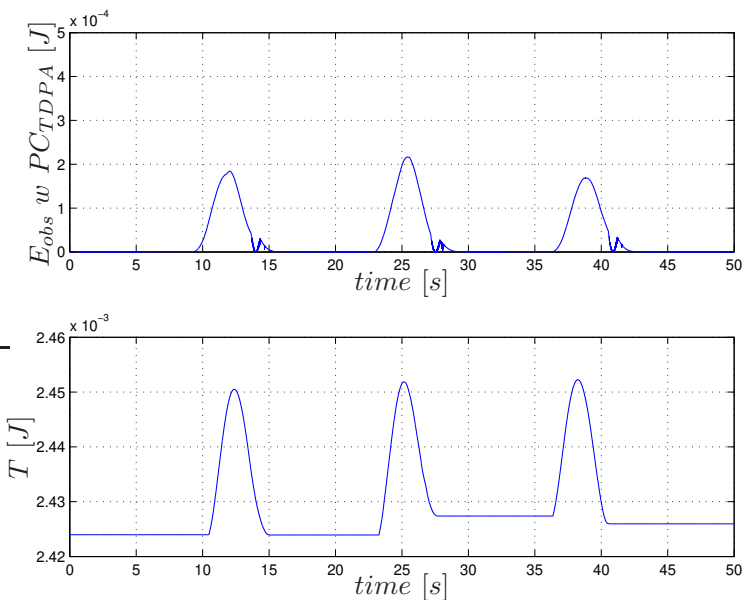

(b) Energy comparison with TDPA (top) and energy of the tank (bottom).

Fig. 11: Experiments results: control input for each method and energy comparison (TDPA and WV).

The experiments with the proposed control can be seen in the video accompanying this paper.

\section{RoBustness ANALYsis}

In this section, an analysis of robustness is performed considering the variation of the time delay in the control loop. The analysis exploits the Montecarlo method. A number of 50 simulations are considered and the time delay changes randomly between $1 \mathrm{~ms}$ and $50 \mathrm{~ms}$. The variation of time delay causes an increase of energy, whose error with respect to the ideal case has mean value $\bar{E}_{t d}=0.5325$ and standard deviation $\sigma_{\bar{E}}=0.0559 \mathrm{~J}$. This is compared in Fig. 12 with the ideal case (no delay) and the energies with the respective energy-based methods, which compensate the time delay effect. In particular, the resulting mean values with the standard deviations $\sigma$ can be found in Table II for the energy and the velocity.

It is worth to notice that the proposed method using energy tank shows the same robustness as the TDPA considering the variation of time delay, as can be seen by the resulting mean value of energy and velocity in Table II. For the

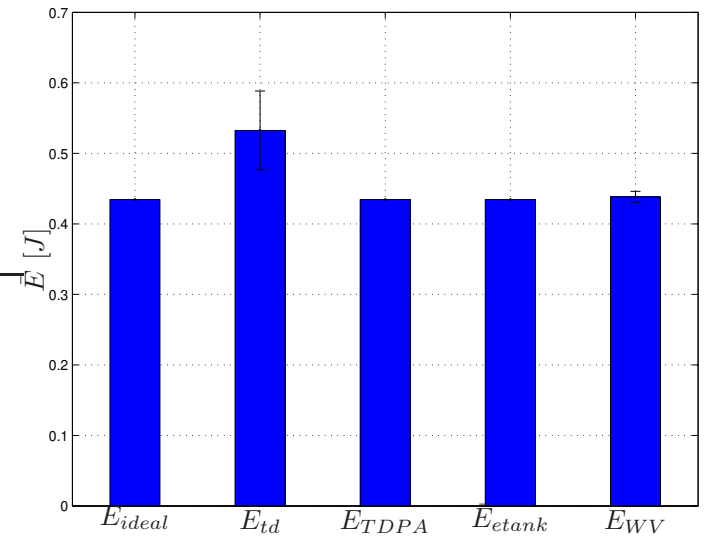

Fig. 12: Robustness analysis. $E_{\text {ideal }}$ is the ideal energy, $\bar{E}_{t d}$ is the energy with time delay in the loop. $\bar{E}_{T D P A}, \bar{E}_{\text {etank }}, \bar{E}_{W V}$ are the respective energy mean value for each method.

\begin{tabular}{|c|c|c|c|c|}
\hline & \multicolumn{4}{|c|}{ Robustness } \\
\hline & \multicolumn{2}{|c|}{ Mean Value } & \multicolumn{2}{c|}{ Standard deviation } \\
\hline & $\dot{\bar{x}}[\mathrm{~m} / \mathrm{s}]$ & $\overline{\bar{E}}[\mathrm{~J}]$ & $\sigma_{\dot{\bar{x}}}$ & $\sigma_{\overline{\bar{E}}}$ \\
\hline Ideal & -0.0071 & 0.4345 & 0 & 0 \\
\hline TDPA & -0.0071 & 0.4345 & 0.00002 & 0.0001 \\
\hline Energy Tank & -0.0071 & 0.4345 & 0.00005 & 0.0002 \\
\hline Wave Variable & -0.0079 & 0.4384 & 0.0031 & 0.0078 \\
\hline
\end{tabular}

TABLE II: Robustness analysis for the considered energy-based methods.

wave variable method, the same problems described in the previous section are found, in particular the overshooting of the velocity affects the behaviour of the system and therefore the mean values in velocity and energy is higher than the ideal case.

\section{A. Discussion}

The limitations and the benefits of each method are summarised in this subsection. The limitation of the wave variable approach is given by the lack of flexibility in parameters setting. In particular, the response of the system depends on the setting of the impedance characteristic $b$. This justifies also the small increase in energy with respect to the ideal case (see Fig. 12) due to the fixed value $b$.

Better performance can be achieved by using the TDPA or the energy tank method. The TDPA method has the advantage that no tuning of parameters is required because the passivity controller dissipates, through a variable damping, the exact amount of active energy monitored by the passivity observer. However, high frequency jitter in velocity might occur, which are generally reported as a limitation of the TDPA damping injection [31].

The proposed method based on energy tank has the flexibility that a generic control action can be chosen. The minimum amount of energy stored in the tank, $\epsilon$ and the control gains need to be set. Then the method relies on some parameters, however, it results to be robust when variable time-delay is considered and it does not generate high frequency jitter in force. 


\section{CONCLUSions AND Future WORKS}

In this paper an energy-based control, which compensates the time delay inherit in the control loop of a robot has been presented. The method allows to simulate free- floating dynamics on an admittance-controlled robot. The approach is based on an energy tank which is exploited by the controller to dissipate the extra energy induced by the time-delay. A robustness analysis and a comparison with different existing methods (TDPA and wave variable) has been performed considering the variation of time delay in the control loop. The results, both in simulations and experiments, show good performance of the proposed method which results robust also to noise intrinsic in the hardware.

Future works aim at extending the method to the multidof case while combining the TDPA control with the energy tank method.

\section{REFERENCES}

[1] J. L. Schwartz, M. A. Peck, and C. D. Hall, "Historical review of airbearing spacecraft simulators," in Journal of Guidance, Control and Dynamics, 2003, pp. AAS 03-125.

[2] A. Flores-Abad, O. Ma, K. Pham, and S. Ulrich, "A review of space robotics technologies for on-orbit servicing," Progress in Aerospace Sciences, vol. 68, pp. 1 - 26, 2014.

[3] O. Ma, J. Wang, M. Sarthak, and M. Liu, "Validation plan for spdm task verification facility," in i-SAIRAS symposium on Artificial Intelligence, Robotics and Automation in Space, Japan 2003.

[4] Z. Milenkovic and C. D'Souza, The Space Operations Simulation Center (SOSC) and Closed-loop Hardware Testing for Orion Rendezvous System Design, ser. Guidance, Navigation, and Control and Co-located Conferences. American Institute of Aeronautics and Astronautics, aug 2012, doi:10.2514/6.2012-5034.

[5] T. Boge, T. Wimmer, O. Ma, and T. Tzschichholz, "EPOS - Using Robotics for RvD Simulation of On-Orbit Servicing Missions," Guidance, Navigation, and Control and Co-located Conferences, aug 2010.

[6] J. Paul, A. Dettmann, B. Girault, J. Hilljegerdes, F. Kirchner, I. Ahrns, and J. Sommer, "INVERITAS: a facility for hardware-in-the-loop long distance movement simulation for rendezvous and capture of satellites and other autonomous objects," Acta Astronautica, vol. 116, pp. 1 24.

[7] J. Artigas, M. De Stefano, W. Rackl, R. Lampariello, B. Brunner, W. Bertleff, R. Burger, O. Porges, A. Giordano, C. Borst, and A. AlbuSchaeffer, "The OOS-SIM: An on-ground simulation facility for on-orbit servicing robotic operations," in Robotics and Automation (ICRA), IEEE International Conference on, May 2015, pp. 2854-2860.

[8] N. Diolaiti, G. Niemeyer, F. Barbagli, and J. Salisbury, "Stability of haptic rendering: Discretization, quantization, time delay, and coulomb effects," Robotics, IEEE Transactions on, vol. 22, no. 2, pp. 256-268, April 2006.

[9] M. De Stefano, "Energy-based control for simulation of multi-body dynamics using robotic facilities," in Ph.D. thesis, Univ. of Modena and Reggio Emilia, March 2019.

[10] P. F. Hokayem and M. W. Spong, "Bilateral teleoperation: an historical survey," in Automatica, 2006, vol. 42, 2006, pp. 2035 - 2057.

[11] M. Franken, S. Stramigioli, S. Misra, C. Secchi, and A. Macchelli, "Bilateral telemanipulation with time delays: A two-layer approach combining passivity and transparency," IEEE Transactions on Robotics, vol. 27, no. 4, pp. 741-756, Aug 2011.

[12] B. Hannaford and J.-H. Ryu, "Time domain passivity control of haptic interfaces," in Robotics and Automation ICRA, 2001 IEEE International Conference on, vol. 2, 2001, pp. 1863-1869.

[13] M. De Stefano, J. Artigas, W. Rackl, and A. Albu-Schaeffer, "Passivity of virtual free-floating dynamics rendered on robotic facilities," in Robotics and Automation (ICRA), 2015 IEEE International Conference on, May 2015, pp. 781-788.
[14] M. De Stefano, J. Artigas, and C. Secchi, "An optimized passivitybased method for simulating satellite dynamics on a position controlled robot in presence of latencies," in 2016 IEEE/RSJ International Conference on Intelligent Robots and Systems (IROS), Oct 2016, pp. $5419-5426$.

[15] M. De Stefano, R. Balachandran, J. Artigas, and C. Secchi, "Reproducing physical dynamics with hardware-in-the-loop simulators: A passive and explicit discrete integrator," in Robotics and Automation (ICRA), 2017 IEEE International Conference on, May 2017, pp. 5899-5906.

[16] M. De Stefano, J. Artigas, and C. Secchi, "A passive integration strategy for rendering rotational rigid-body dynamics on a robotic simulator," in 2017 IEEE/RSJ International Conference on Intelligent Robots and Systems (IROS), Sept 2017, pp. 2806-2812.

[17] G. Niemeyer and J. . E. Slotine, "Stable adaptive teleoperation," IEEE Journal of Oceanic Engineering, vol. 16, no. 1, pp. 152-162, Jan 1991.

[18] _ - "Using wave variables for system analysis and robot control," in Proceedings of International Conference on Robotics and Automation, vol. 2, April 1997, pp. 1619-1625 vol.2.

[19] C. Secchi, F. Ferraguti, and C. Fantuzzi, "Catching the wave: A transparency oriented wave based teleoperation architecture," in 2016 IEEE International Conference on Robotics and Automation (ICRA), May 2016, pp. 2422-2427.

[20] F. Ferraguti, C. T. Landi, L. Sabattini, M. Bonfè, C. Fantuzzi, and C. Secchi, "A variable admittance control strategy for stable physical human-robot interaction," The International Journal of Robotics Research, vol. 38, no. 6, pp. 747-765, 2019. [Online]. Available: https://doi.org/10.1177/0278364919840415

[21] F. Ferraguti, N. Preda, A. Manurung, M. Bonfè, O. Lambercy, R. Gassert, R. Muradore, P. Fiorini, and C. Secchi, "An energy tankbased interactive control architecture for autonomous and teleoperated robotic surgery," IEEE Transactions on Robotics, vol. 31, no. 5, pp. 1073-1088, Oct 2015.

[22] C. T. Landi, F. Ferraguti, L. Sabattini, C. Secchi, M. Bonfè, and C. Fantuzzi, "Variable admittance control preventing undesired oscillating behaviors in physical human-robot interaction," in 2017 IEEE/RSJ International Conference on Intelligent Robots and Systems (IROS), Sept 2017, pp. 3611-3616.

[23] I. Aliaga, A. Rubio, and E. Sanchez, "Experimental quantitative comparison of different control architectures for master-slave teleoperation," IEEE Transactions on Control Systems Technology, vol. 12, no. 1, pp. 2-11, Jan 2004.

[24] P. Arcara and C. Melchiorri, "Control schemes for teleoperation with time delay: A comparative study," Robotics and Autonomous Systems, vol. 38, no. 1, pp. 49 - 64, 2002. [Online]. Available: http://www.sciencedirect.com/science/article/pii/S0921889001001646

[25] R. Balachandran, J. Artigas, U. Mehmood, and J. Ryu, "Performance comparison of wave variable transformation and time domain passivity approaches for time-delayed teleoperation: Preliminary results," in 2016 IEEE/RSJ International Conference on Intelligent Robots and Systems (IROS), Oct 2016, pp. 410-417.

[26] B. Siciliano and O. Khatib, Eds., Springer Handbook of Robotics. Springer, 2008.

[27] A. van der Schaft, $L_{2}$-Gain and Passivity Techniques in Nonlinear Control, ser. Communication and Control Engineering. Springer, 2000.

[28] J.-H. Ryu, D.-S. Kwon, and B. Hannaford, "Stability guaranteed control: Time domain passivity approach," IEEE Transactions on Control Systems Technology, vol. 12, no. 6, pp. 860-868, 2004.

[29] C. Secchi, S. Stramigioli, and C. Fantuzzi, Control of Interactive Robotic Interfaces: a port-Hamiltonian Approach, ser. Springer Tracts in Advanced Robotics. Springer, 2007.

[30] G. Niemeyer and J. J. Slotine, "Telemanipulation with Time Delays," International Journal of Robotics Research, vol. 23, no. 9, pp. 873890, September 2004.

[31] Y. S. Kim and B. Hannaford, "Some practical issues in time domain passivity control of haptic interfaces," in Proceedings 2001 IEEE/RSJ International Conference on Intelligent Robots and Systems. (Cat. No.01CH37180), vol. 3, 2001, pp. 1744-1750 vol.3. 